\title{
What makes a good review from an editor's perspective?
}

\author{
Gérard Hopfgartner ${ }^{1}$
}

Published online: 29 September 2017

(C) Springer-Verlag GmbH Germany 2017

One major aim of scientific publishing is to share knowledge to a larger audience; this indirectly contributes in various ways to the improvement of society. It also provides the authors with positive visibility in the scientific community. The report of novel scientific information or the reviewing of state-ofthe-art knowledge is a complex endeavor, in which, in addition to the content, ethics play a major role in calling for standardization. In that regard, editors forums, such as the Committee on Publishing Ethics (COPE), are an important platform to share best practices and provide guidelines [1]. Apart from the journal scope and recommendation to the authors of the journal, reviewers can also find additional information on COPE regarding best practices.

In the first step of the publishing process, when a manuscript is submitted to the journal, it is assigned to an editor with the required expertise. The manuscript will be screened for content originality compared to published work using informatics tools, such as iThenticate [2]. In the second step, the editor has to decide if the manuscript is addressing the scope of the journal or not, and if the answer is positive the editor looks for suitable reviewers. The review process is essential to assess the originality of the work and the quality of the reporting. Based on the reviewers' reports, the editor makes a decision: accept without modification, minor revision, major revision, reject with possible resubmission, or reject. The aim of the process is to be as fair as possible in a short timeframe. Of course, the evaluation of a peer's research can be a challenging and subjective task. It often results in two different

Gérard Hopfgartner

gerard.hopfgartner@unige.ch

1 Life Sciences Mass Spectrometry, Department of Analytical and Inorganic Chemistry, University of Geneva, 24 Quai Ernest Ansermet, CH-1211 Geneva 4, Switzerland opinions and the editor has either to request an additional reviewer or make a decision based on provided comments. Two oft-seen reviews are "Interesting manuscript should be published as is" or "This is a poor manuscript, which should be rejected because the topic and the data presented are not novel"; both without any additional comments. From an editor's perspective, these are not very useful reviews. Besides these very short reviews, one also receives reviews of four to five pages of comments, including extensive typo and grammar corrections, and suggestions of additional experiments. Extensive review can help to improve the manuscript, but too many comments may also result in significant confusion for the author. So, what is the right balance and what should a reviewer keep in mind? The first goal of a review is to evaluate the science presented. Acceptance or rejection should be primarily based on the originality and quality of the work, and the reviewer's role is to provide statements supporting the strengths and weaknesses of the manuscript. The question of evaluating novelty is clearly challenging because two categories of papers need to be taken into account: (1) novel instrumentation or workflows, and (2) novel applications. Here the reviewer should ask (i) are the data presented in the paper supporting the claims made by the authors and (ii) is the work novel with regard to published papers?

A very well-written and organized paper can be rejected because it does not present significant analytical novelty. One typical case is the quantification of analytes in biological fluids, such as plasma, based on protein precipitation using liquid chromatography tandem mass spectrometry or a metabolomics study using multivariate analysis without any compound identification.

The rejection of a manuscript does not mean that it is the end of it, but it means that it has not reached the expected standards of the journal. The authors always have the possibility of submitting their work to another journal or 
resubmitting it to the same journal. In any case, a revision would be required; this is why a reviewer's negative comments should always be scientifically informative.

Another important point reviewers should keep in mind are the cited references. In the introduction and in the results and discussion sections, the reviewer should check that relevant publications to the topic are referenced. Adequate referencing enables the less specialized or younger readers to get the complete picture of a research theme. Unfortunately, in rare cases, self-citation is predominant or some authors forget to cite their similar work published in other journals. The editor very much relies on the expertise of the reviewers to identify these type of issues.

A request of revision (minor or major) should help to improve the presentation/readability of the work. Sometimes the scientific content is original, but the content of the manuscript is not sufficiently strong. In this case, a reject/resubmission should be considered to give the authors sufficient time to complete their work.

So, in summary, what is the best review from an editor's perspective? Remember that any scientist has two hats: as an author and a reviewer in serving the community. A review does not need to be extremely detailed, but the decision should be supported by informative statements about strength and weakness of the manuscript in its content and presentation. As an editor, I am grateful for the reviewer's time and expertise, and the important role they play in the publishing process and in the advancement of scientific knowledge.

\section{References}

1. https://publicationethics.org/files/Ethical_guidelines_for_peer reviewers_0.pdf, consulted September 09, 2017.

2. Butler D. Journals step up plagiarism policing. Nature. 2010;466(7303):167. https://doi.org/10.1038/466167a.

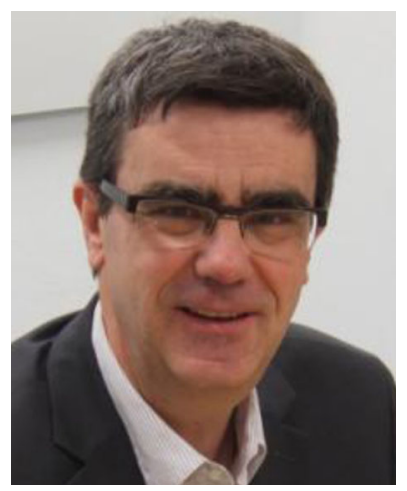

Gérard Hopfgartner is Professor for analytical chemistry and mass spectrometry in the Department of Analytical and Inorganic Chemistry at the University of Geneva in Geneva, Switzerland. His research interests focus on the application and development of novel hyphenated mass spectrometry approaches in the field of life sciences from elements to proteins. His current efforts includes separation sciences, sample preparation, bioanalysis, drug metabolism, metabolomics, analytical proteomics, toxicology, high-resolution mass spectrometry, ion mobility mass spectrometry, and mass spectrometry imaging. He has been Editor of Analytical and Bioanalytical Chemistry (ABC) since 2016. 